\title{
Heme crystallization in the midgut of triatomine insects ${ }^{\text {th }}$
}

\author{
Marcus F. Oliveira ${ }^{a, *}$, Ana Caroline P. Gandara ${ }^{a}$, Cláudia M.S. Braga ${ }^{b}$, José R. Silva ${ }^{c}$, \\ Flavia B. Mury ${ }^{c}$, Marílvia Dansa-Petretski ${ }^{c}$, Diego Menezes ${ }^{\text {d }}$, \\ Marcos A. Vannier-Santos ${ }^{d}$, Pedro L. Oliveira ${ }^{a}$ \\ a Instituto de Bioquímica Médica, Programa de Biotecnologia e Biologia Molecular, Universidade Federal do Rio de Janeiro, \\ Cidade Universitária, Rio de Janeiro, RJ, 21941-590, Brazil \\ b Petrobrás/CENPES, Divisão de Química, Setor de Química Orgânica, Cidade Universitária, Rio de Janeiro, RJ, 21949-900, Brazil \\ ${ }^{c}$ Centro de Biociências e Biotecnologia, Universidade Estadual do Norte Fluminense, Campos dos Goytacazes, RJ, 28013-602, Brazil \\ ${ }^{\mathrm{d}}$ Centro de Pesquisas Gonçalo Moniz, Fiocruz, Salvador, BA, Brazil
}

Received 13 April 2006; received in revised form 26 October 2006; accepted 8 December 2006

Available online 19 December 2006

Dedicated to the memory of Carmelita Mendes de Oliveira (1913-2006)

\begin{abstract}
Hemozoin $(\mathrm{Hz})$ is a heme crystal produced by several blood-feeding organisms in order to detoxify free heme released upon hemoglobin (Hb) digestion. Here we show that heme crystallization also occurs in three species of triatomine insects. Ultraviolet-visible and infrared light absorption spectra of insoluble pigments isolated from the midgut of three triatomine species Triatoma infestans, Dipetalogaster maximus and Panstrongylus megistus indicated that all produce Hz. Morphological analysis of T. infestans and D. maximus midguts revealed the close association of $\mathrm{Hz}$ crystals to perimicrovillar membranes and also as multicrystalline assemblies, forming nearly spherical structures. Heme crystallization was promoted by isolated perimicrovillar membranes from all three species of triatomine bugs in vitro in heat-sensitive reactions. In conclusion, the data presented here indicate that $\mathrm{Hz}$ formation is an ancestral adaptation of Triatominae to a blood-sucking habit and that the presence of perimicrovillar membranes plays a central role in this process.
\end{abstract}

(C) 2007 Elsevier Inc. All rights reserved.

Keywords: Triatominae; Heme; Hemozoin; Perimicrovillar membranes; Midgut

\section{Introduction}

A great variety of organisms, from protozoa to mammals, uses the vertebrate blood as the main food source. Hemoglobin $(\mathrm{Hb})$ is a major blood component and during its digestion, in the

\footnotetext{
This paper is part of the 4th special issue of CBP dedicated to The Face of Latin American Comparative Biochemistry and Physiology organized by Marcelo Hermes-Lima (Brazil) and co-edited by Carlos Navas (Brazil), Rene Beleboni (Brazil), Rodrigo Stabeli (Brazil), Tania Zenteno-Savín (Mexico) and the editors of CBP. This issue is dedicated to the memory of two exceptional men, Peter L. Lutz, one of the pioneers of comparative and integrative physiology, and Cicero Lima, journalist, science lover and Hermes-Lima's dad.

* Corresponding author. Instituto de Bioquímica Médica, Programa de Biologia Molecular e Biotecnologia, Universidade Federal do Rio de Janeiro, Cidade Universitária, Rio de Janeiro, RJ, 21941-590, Brazil. Tel.: +55 21 25626755; fax: +552122708647

E-mail address: maroli@bioqmed.ufrj.br (M.F. Oliveira).
}

digestive tract of blood-feeding organisms, an intense release of peptides, amino acids and heme takes place (Francis et al., 1997; Brindley et al., 1997). Despite the obvious biological role, once in a free state heme acts as potent cytotoxic agent, promoting the lysis of many cell types (Chou and Fitch, 1980; Orjih et al., 1981 ) and also the generation of reactive oxygen species (ROS) through catalytic decomposition of organic hydroperoxides (Davies, 1988; Van der Zee et al., 1996). Heme-mediated-ROS generation leads to oxidative stress which could result in damage of biomolecules such as proteins, carbohydrates and lipids (Tappel, 1955; Vincent, 1989). Furthermore, at millimolar concentrations, heme can associate with cellular phospholipid membranes, reducing its degree of order and increasing its permeability (Schmitt et al., 1993). Thus, the way in which blood-feeding organisms deal with free heme is of central importance to their physiologies. 
T. infestans

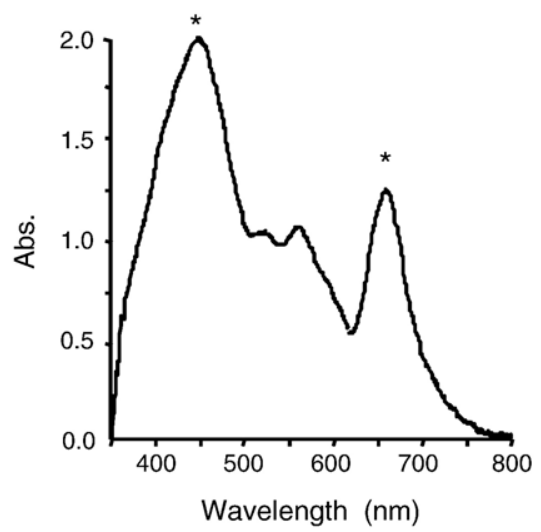

P. megistus

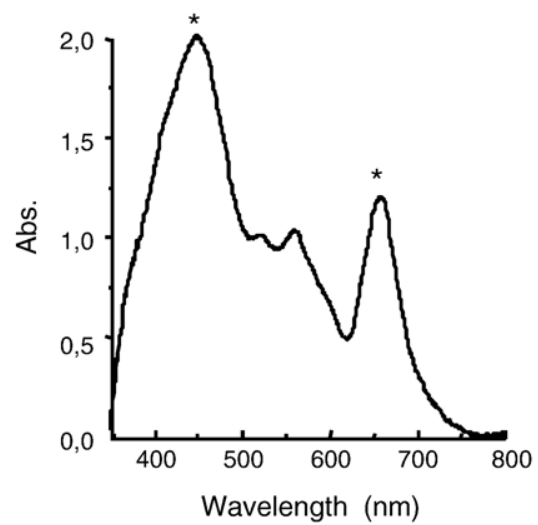

D. maximus
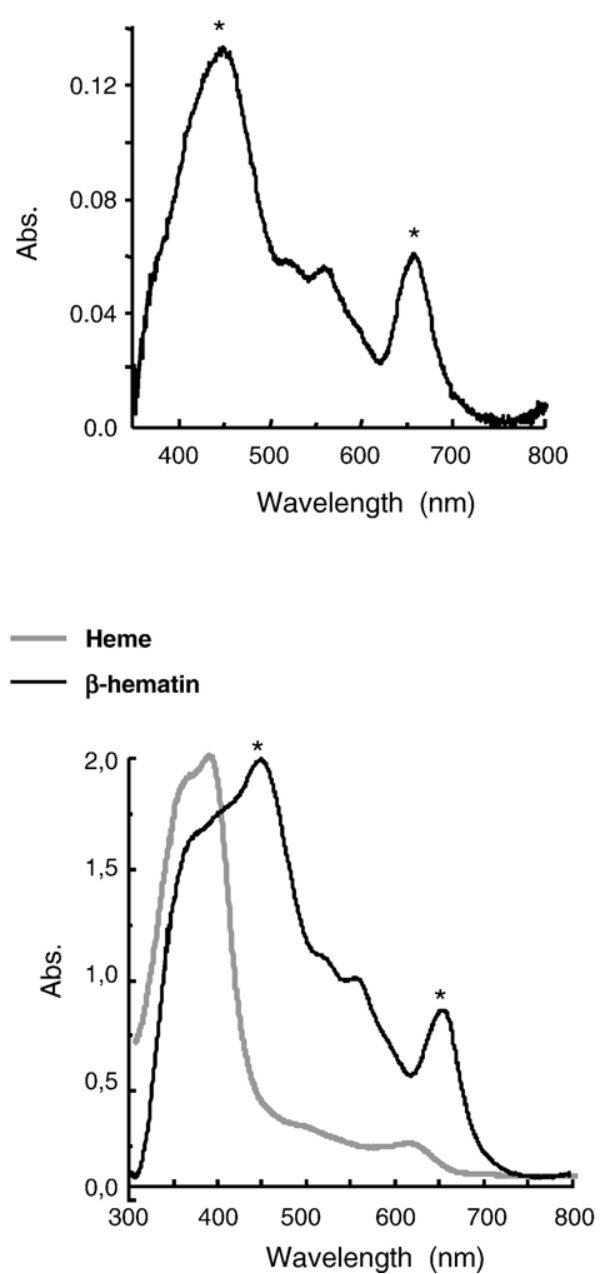

Fig. 1. UV-visible spectroscopic analysis of heme pigments in the midgut of three triatomine species. The midgut of blood-fed Triatoma infestans, Dipetalogaster maximus and Panstrongylus megistus insects were subjected to the Hz extraction protocol as described in the Materials and methods section. The obtained pigments were resuspended in $0.1 \mathrm{M} \mathrm{NaHCO}_{3}, \mathrm{pH} 9.1$ and analyzed by UV-visible spectrophotometry. Analysis of commercial hemin (gray line) and in vitro synthesized $\beta$-hematin were also carried out. The distinctive Hz peaks in all spectra were indicated by asterisks.

In order to overcome heme toxicity, very efficient mechanisms of detoxification have evolved in such organisms (Graça-Souza et al., 2006), including the enzyme heme oxygenase (Paiva-Silva et al., 2006) and heme binding proteins from the hemolymph of the cattle tick Boophilus microplus (Maya-Monteiro et al., 2004) and from Rhodnius prolixus, a vector of Chagas' disease (DansaPetretski et al., 1995). In malaria parasites, $\mathrm{Hb}$ digestion occurs inside the digestive vacuole, followed by crystallization of free heme into a dark brown pigment named hemozoin $(\mathrm{Hz})$ (Slater et al., 1991; Pagola et al., 2000). Besides malaria parasites, heme crystallization was also reported in $R$. prolixus and in the helminth Schistosoma mansoni, representing one of the major heme detoxification pathways in these organisms (Oliveira et al., 1999, 2000a,b, 2002, 2004). Moreover, $\mathrm{Hz}$ was also found in both the avian protozoan Haemoproteus columbae and in the rediae of the trematode Echinostoma trivolvis (Chen et al., 2001; Pisciotta et al., 2005). Concerning the mechanism of heme crystallization, in $R$. prolixus we showed that the perimicrovillar membranes, which are phospholipid bilayer membranes that cover the epithelial midgut cells (Lane and Harrison, 1979; Gutierrez and Burgos, 1986), were capable to promote heme crystallization in vitro (Oliveira et al., 2000a). Moreover, $\mathrm{Hz}$ crystals were found both associated to vesicles derived from perimicrovillar membranes and also free in the midgut lumen or even in multicrystalline assemblies, suggesting that hydrophobic environments and/ or protein components of these membranes played an essential role in heme crystallization (Oliveira et al., 2005). Thus, since the presence of perimicrovillar membranes is a characteristic feature of all hemipterans, here we investigated whether heme crystallization could also occur in different species of the triatomine vectors of Chagas' disease.

\section{Materials and methods}

\subsection{Chemicals and reagents}

Hemin chloride was purchased from Sigma Chemicals (St. Louis, MO, USA). All other reagents were of analytical grade. 
T. infestans

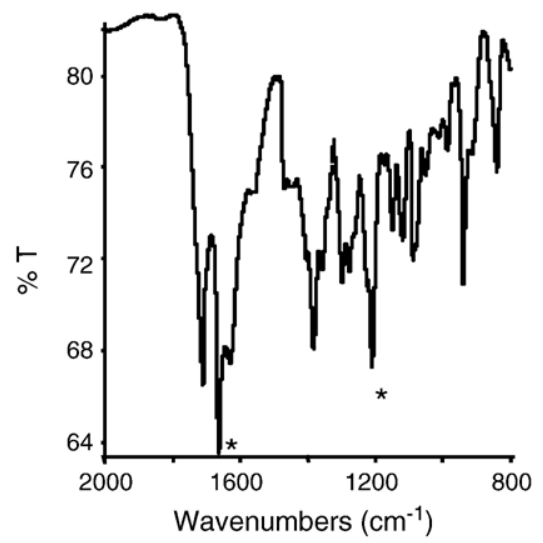

D. maximus

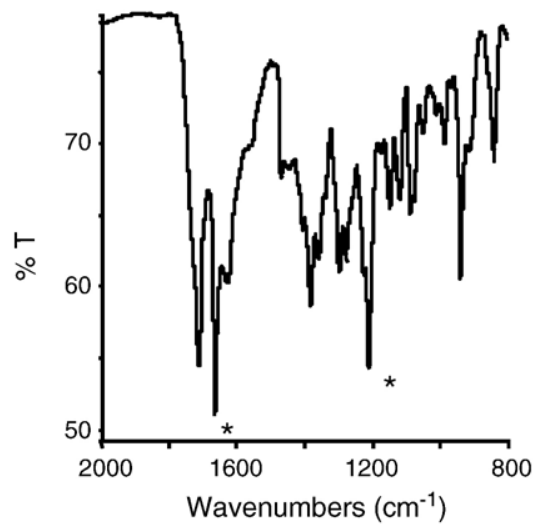

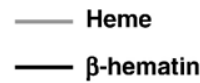

P. megistus

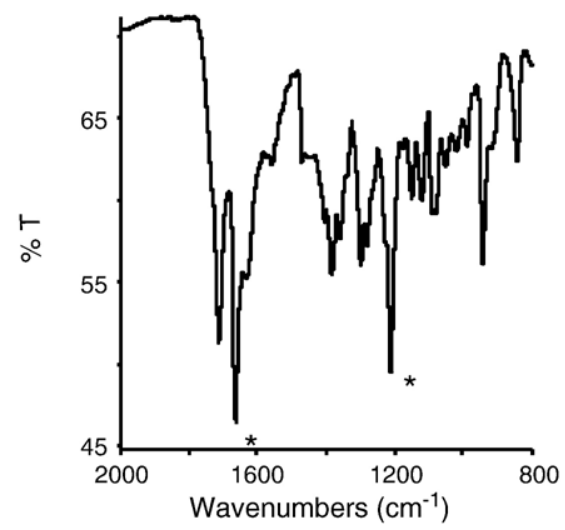

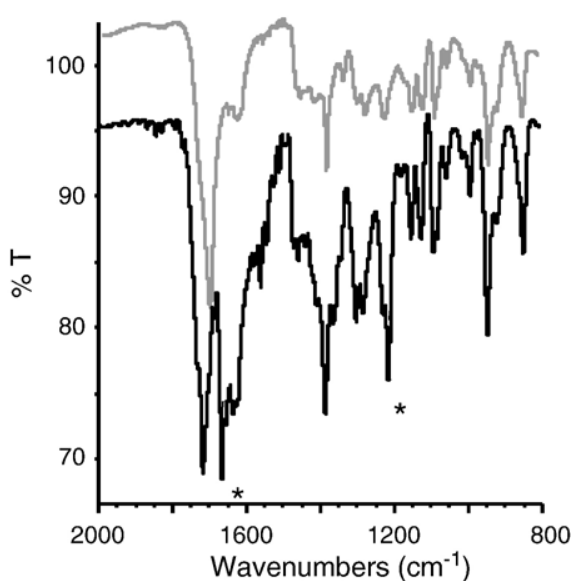

Fig. 2. FTIR spectroscopic analysis of heme pigments in the triatomine midgut. The midgut of blood-fed Triatoma infestans, Dipetalogaster maximus and Panstrongylus megistus insects were subjected to the $\mathrm{Hz}$ extraction protocol as described in the materials and methods section. $\mathrm{KBr}$ pellets of the obtained material were analyzed by Fourier-transformed infrared spectroscopy (FTIR). Analysis of commercial hemin (gray line) and in vitro synthesized $\beta$-hematin were also carried out. The distinctive Hz peaks in FTIR spectra are indicated by asterisks.

\subsection{Animals}

Blood-fed adult females of Triatoma infestans, Dipetalogaster maximus and Panstrongylus megistus were obtained from Laboratório Nacional e Internacional de Referência em Taxonomia de Triatomíneos at Departamento de Entomologia, Fundação Oswaldo Cruz, Rio de Janeiro, Brazil. R. prolixus adult females were reared with rabbit blood and kept for four days at $28^{\circ} \mathrm{C}$ and $80 \%$ relative humidity.

\subsection{Hz extraction and spectroscopic analysis}

$\mathrm{Hz}$ was extracted from $T$. infestans, D. maximus and $P$. megistus based on a method previously described for R. prolixus (Oliveira et al., 1999). Midgut contents from these bugs were obtained by gently shaking the dissected midguts in $0,15 \mathrm{M} \mathrm{NaCl}$. Tissue was discarded and the suspension was centrifuged at $15,000 \times g$ for $20 \mathrm{~min}$. The insoluble pigment was further purified by three washes with $0.1 \mathrm{M} \mathrm{NaHCO}_{3}+$
$2.5 \% \mathrm{SDS}, \mathrm{pH} 9,1$. The remaining solids were washed twice with water and kept at $4{ }^{\circ} \mathrm{C}$ until analysis. Spectrophotometric determinations were carried out by resuspending the pellets in $0.1 \mathrm{M} \mathrm{NaHCO}_{3} \mathrm{pH} 9.1$ in GBC/UV-920 spectrophotometer. For infrared absorption experiments, $\mathrm{KBr}$ pellets were prepared from dried samples of each pigment and spectra were acquired for 32 cycles with a FTIR spectrometer (Nicolet, Magna 550).

\subsection{Heme crystallization in vitro}

Midgut samples from blood-fed insects were obtained and stored in $0.15 \mathrm{M} \mathrm{NaCl}$ containing $0.5 \mathrm{mM}$ benzamidine, $50 \mu \mathrm{g} /$ $\mathrm{mL}$ soybean trypsin inhibitor, $0.02 \mathrm{mg} / \mathrm{mL}$ antipain, $10 \mu \mathrm{M}$ pepstatin, $0.1 \mathrm{mM} \mathrm{Zn}$ acetate and $2 \mathrm{mM}$ dithiothreitol. After homogenization, samples were kept at $-70^{\circ} \mathrm{C}$ until use. Later this material was centrifuged at $15,000 \times g$ for $20 \mathrm{~min}$ at $5{ }^{\circ} \mathrm{C}$ and the amount of protein in the pellet was measured (Lowry et al., 1951). The capacity of these fractions to promote heme crystallization was evaluated as previously described (Oliveira et al., 2000a). 


\section{T. infestans}

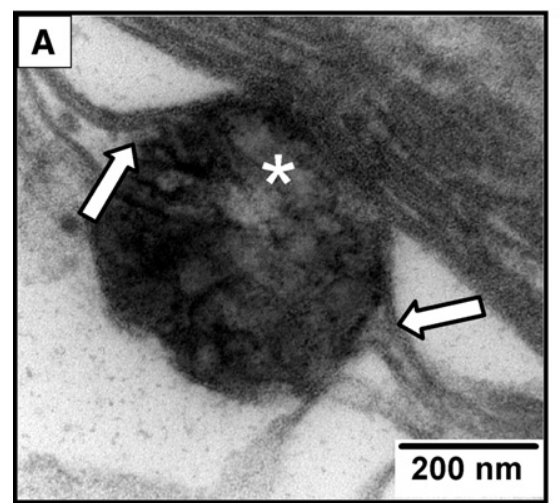

\section{D. maximus}

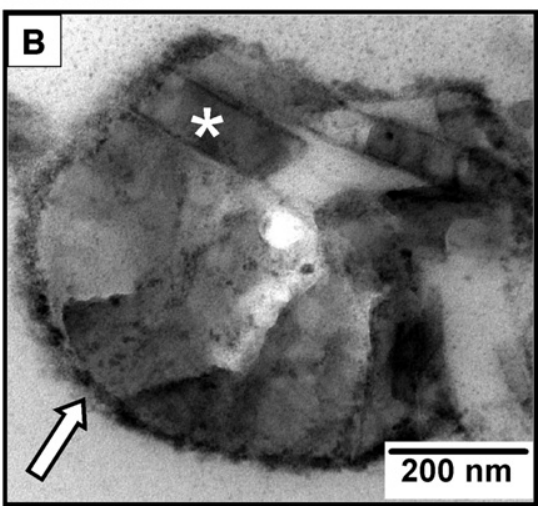

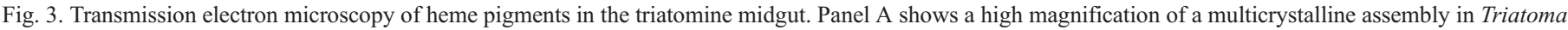

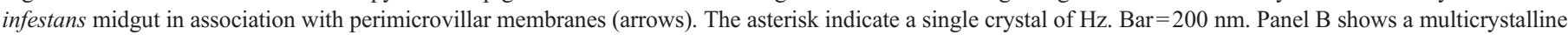

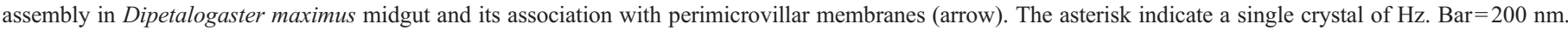

Briefly, samples of $20 \mu \mathrm{g}$ of protein were incubated for $24 \mathrm{~h}$ at $28^{\circ} \mathrm{C}$ in $0.5 \mathrm{M}$ sodium acetate, $\mathrm{pH} 4.8$, in the presence of $100 \mu \mathrm{M}$ hemin. After incubation, the reaction mixture was centrifuged $15,000 \times g$ for $20 \mathrm{~min}$ at $25^{\circ} \mathrm{C}$. The pellet was washed three times with $1 \mathrm{~mL}$ of $0.1 \mathrm{M} \mathrm{NaHCO}_{3}+2.5 \% \mathrm{SDS}, \mathrm{pH} 9.1$, and twice with deionized water. The final pellet was solubilized in $0.1 \mathrm{M} \mathrm{NaOH}$ and the amount of heme determined spectrophotometrically at $400 \mathrm{~nm}$ in a GBC-UV/Vis-920 spectrophotometer.

\subsection{Transmission electron microscopy (TEM)}

Midguts from blood-fed T. infestans and D. maximus were fixed overnight at room temperature in $1 \%$ glutaraldehyde and $4 \%$ formaldehyde in $0.1 \mathrm{M}$ sodium cacodylate buffer, $\mathrm{pH}$ 7.4. They were then rinsed and postfixed in $1 \% \mathrm{OsO}_{4}, 0.8 \% \mathrm{~K}_{3} \mathrm{Fe}(\mathrm{CN})_{6}$ and $5 \mathrm{mM} \mathrm{CaCl}_{2}$ in the same buffer for $1 \mathrm{~h}$. Samples were dehydrated in acetone and embedded in epoxy Polybed resin (Polyscience). Ultrathin sections were stained with uranyl acetate, lead citrate and were observed in a Zeiss 109 electron microscope. Images were acquired using a Megaview II digital system.

\section{Results}

Since we identified $\mathrm{Hz}$ in the midgut of the triatomine insect $R$. prolixus our first approach was to investigate the presence of similar pigments in three other species of triatomine insects. So, using methods previously described for isolation of $R$. prolixus $\mathrm{Hz}$ (Oliveira et al., 1999), we obtained dark-brown insoluble pellets from midgut samples of blood-fed $T$. infestans, D. maximus and P. megistus. Fig. 1 shows the UV-visible absorption spectra of the intact pigments, which were markedly distinct from the absorption of heme (gray line) and very similar to that of $\beta$-hematin, Plasmodium $\mathrm{Hz}$ (Fitch and Kanjananggulpan, 1987), R. prolixus $\mathrm{Hz}$ (Oliveira et al., 2000a) and $S$. mansoni $\mathrm{Hz}$ (Oliveira et al., 2000b). These spectra share some characteristic features such as a broad Soret absorbance band centered near $450 \mathrm{~nm}$ and also another peak near $660 \mathrm{~nm}$. These pigments were insoluble in neutral and slightly alkaline ( $\mathrm{pH}$ 9.1) solutions but were readily dissolved in $0.1 \mathrm{M}$ sodium hydroxide, a condition that changed their light absorption spectra to that typical of monomeric heme (data not shown). Fourier transform infrared spectroscopy (FTIR) of intact pigments also revealed spectra similar to what was described for $\mathrm{Hz}$ from Plasmodium, R. prolixus and S. mansoni. Specific transmittance peaks near $1210 \mathrm{~cm}^{-1}$ and $1663 \mathrm{~cm}^{-1}$ indicate the presence of iron-carboxylate bonds responsible for $\mathrm{Hz}$ structure (Fig. 2 and Slater et al., 1991). Taken together, these results undoubtedly demonstrate that the triatomines T. infestans, D. maximus and P. megistus produce $\mathrm{Hz}$ in their midguts as an efficient way to detoxify heme.

We have recently shown heme crystals produced in Schistosoma and Rhodnius guts assemble themselves as multicrystalline structures composed of long regular brick-shaped crystals of approximately $200 \mathrm{~nm}$ length, forming large spherical structures (Oliveira et al., 2005). In contrast, Hz crystals in malaria parasites are arranged in a distinct pattern without the formation of

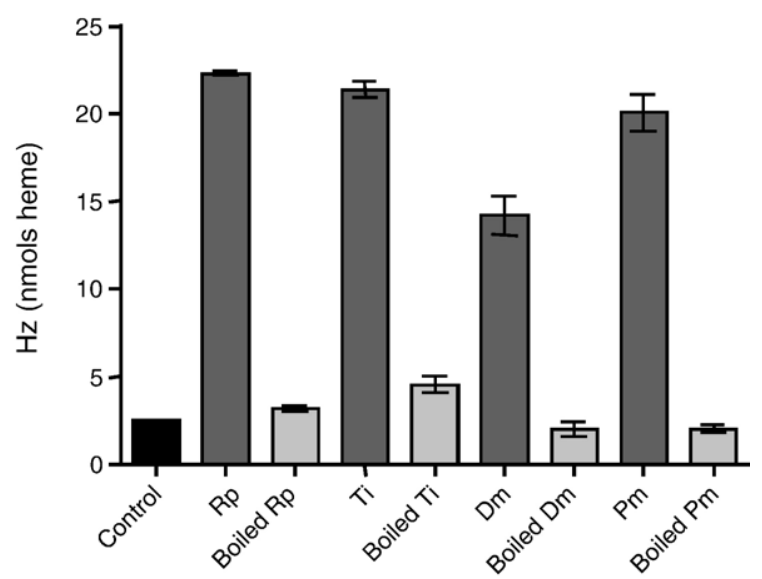

Fig. 4. Heme crystallization activity of triatomine perimicrovillar membranes. Dark grey bars represent samples of midgut membranes ( $20 \mu \mathrm{g}$ of protein) from blood-fed Rhodnius prolixus (Rp), Triatoma infestans (Ti), Dipetalogaster maximus (Dm) and Panstrongylus megistus (Pm) were assayed for heme crystallization activity as described under methods. Light grey bars represent samples of midgut membranes $\left(20 \mu \mathrm{g}\right.$ of protein) pre-incubated at $100{ }^{\circ} \mathrm{C}$ for $30 \mathrm{~min}$, then assayed for heme crystallization activity. The black bar represents the product of heme crystallization in the absence of the triatomine midgut membranes. After incubation $\mathrm{Hz}$ was extracted following methods described in the literature. All results shown are expressed as mean \pm S.E.M. $(n=4)$. 
regular multicrystalline assemblies. The ultrastructural investigation of T. infestans and D. maximus midguts by transmission electron microscopy (TEM) revealed the presence of these multicrystalline assemblies of $\mathrm{Hz}$ in these two triatomine insects, just like those found in R. prolixus and in S. mansoni (Fig. 3, asterisks). Moreover, a close association of the multicrystalline assemblies to perimicrovillar membranes was also observed in both triatomine insects (Fig. 3, arrows). This prompted us to determine whether the perimicrovillar membranes of these three species of triatomine species promote heme crystallization, as we described in Rhodnius. In fact, preparations enriched in perimicrovillar membranes isolated from $T$. infestans, $D$. maximus and $P$. megistus midguts were all capable to promote $\mathrm{Hz}$ formation in vitro (Fig. 4). Interestingly, heme crystallization activities, induced by these membranes, were all sensitive to previous heating to $100{ }^{\circ} \mathrm{C}$ indicating that this activity is similar to that of Rhodnius (Oliveira et al., 2000a) and distinct from Plasmodium and Schistosoma, where boiling caused no changes in their abilities to promote $\mathrm{Hz}$ formation (Dorn et al., 1995; Oliveira et al., unpublished data).

\section{Discussion}

Increased levels of free heme inside the gut of blood feeders poses a challenge to these organisms, which usually ingest large amounts of $\mathrm{Hb}$, due to the intrinsic heme toxicity (Ryter and Tyrrel, 2000). To avoid this, blood feeders developed several adaptations (Graça-Souza et al., 2006) such as precipitation of free heme inside their digestive tract through its crystallization into hemozoin $(\mathrm{Hz})$, a process that represents the quantitatively most important heme detoxification pathway of these organisms (Slater et al., 1991; Pagola et al., 2000; Egan et al., 1994; Oliveira et al., 1999, 2000a,b, 2002, 2004; Graça-Souza et al., 2006). This mechanism is essential for Plasmodium survival as more than $95 \%$ of the heme iron released from host $\mathrm{Hb}$ is incorporated into $\mathrm{Hz}$ in food vacuoles (Egan et al., 2002). Inhibition of $\mathrm{Hz}$ formation has been shown to induce biological damage in both Rhodnius (Oliveira et al., 2000a) and Schistosoma (Oliveira et al., 2004). In the present work, we demonstrate that true $\mathrm{Hz}$ is found in the midgut of three species of triatomine insects - that, together with Rhodnius, are representative of the most important genera of this subfamily for Chagas disease transmission - and showed that perimicrovillar membranes play a key role in $\mathrm{Hz}$ formation in these insects.

Concerning evolution of hematophagy, there is a dispute about how old is the adaptation to a blood-feeding way of life in the subfamily Triatominae. Some authors have suggested that hematophagy is a monophyletic trait that appeared early in the origin of triatomine reduvids in the American continent in the late cretaceous, about 65 MYA (Gaunt and Miles, 2000). In contrast, Schofield (2000) has advocated the hypothesis that - in spite of the antiquity of the origin of the group - hematophagy is a comparatively recent behaviour, initiated less than 5 MYA and having evolved independently in the tribes Rhodniini and Triatomini. The fact that the capacity to promote $\mathrm{Hz}$ formation is widespread across the subfamily adds evidence in favour of a monophyletic origin of blood feeding. $\mathrm{Hz}$ formation represents therefore an important adaptation that allows digestion of large amounts of blood and our evidence indicates a role for perimicrovillar membranes in heme crystallization in the midgut of Triatominae. As all hemipteran insects have perimicrovillar membranes, its presence in the midgut of the non-hematophagous reduvid ancestor could be regarded as a pre-adaptation of those insects that would have alleviated heme deleterious effects and opened the way to develop in the direction of hematophagy, a common feature of all present day triatomine bugs.

The ultrastructural organization of $\mathrm{Hz}$ crystallites in these three triatomine species is quite similar to that of $S$. mansoni and R. prolixus $\mathrm{Hz}$ but distinct from those found in Plasmodium $\mathrm{Hz}$ (Fig. 3; Hempelmann et al., 2003; Oliveira et al., 2005). Likewise, the heat-sensitivity of heme crystallization activity of triatomine perimicrovillar membranes shown here (Fig. 4) resembles the activity found in Rhodnius (Oliveira et al., 2000a) and differ from that present in Schistosoma (Oliveira et al., unpublished data), probably due to intrinsic features of the structures involved in $\mathrm{Hz}$ formation in the guts of these organisms. In S. mansoni, $\mathrm{Hz}$ is produced at the surface of extracellular lipid droplets present in their gut lumen (Oliveira et al., 2005) showing a heat-resistant heme crystallization activity, as would be expected for lipiddriven $\mathrm{Hz}$ formation (Oliveira MF, unpublished data). Mechanistically, the heat sensitivity of heme crystallization activity associated to perimicrovillar membranes suggests the participation of a protein in this process (Slater and Cerami, 1992). The only known protein described to date that would be involved in heme crystallization is the histidine-rich protein-II (HRP-II) in Plasmodium (Sullivan et al., 1996). However, parasites lacking the HRP-II or III genes are still capable to produce $\mathrm{Hz}$ (Sullivan, 2002). Therefore, the role of a specific protein as a physiological promoter of $\mathrm{Hz}$ formation remains elusive. As $\mathrm{Hz}$ formation takes place in close association with hydrophobic structures, such as the food vacuole membranes in Plasmodium (Hempelmann et al., 2003), the perimicrovillar membranes in triatomines (Figs. 3 and 4; Oliveira et al., 2000a) and lipid droplets in Schistosoma (Oliveira et al., 2005), it is tempting to propose that lipids by themselves would be capable to promote heme crystallization into Hz. In line with this possibility, it has been shown that incubation of heme with several compounds such as acetate (Egan et al., 2001), ethanol (Blauer and Akkawi, 2002), benzoic acid (Egan, 2002) and even dimethylsulfoxide (Oliveira MF, unpublished data) increased heme crystallization through solubilization of heme in acidic environments. The increment of heme solubility would occur through displacement of the axial water molecule bound to the central heme iron, thus by-passing the rate-limiting step of the process that is the rapid precipitation of heme (Egan et al., 2001). This is followed by slow conversion to crystalline $\mathrm{Hz}$, in a process induced by phase transfer catalysts, a role that could be performed by lipids in Plasmodium, Schistosoma and in triatomines as well (Bendrat et al., 1995; Dorn et al., 1998; Fitch et al., 1999; Hempelmann et al., 2003; Jackson et al., 2004; Oliveira et al., 2000a, 2004, 2005). In fact, recent data from the literature demonstrated that under physiological conditions, heme crystallization was induced rapidly and spontaneously in vitro near long chain alcohol/water and lipid/water interfaces (Egan et al., 2006). Pisciotta and colleagues have also shown that neutral lipids 
wrapping $\mathrm{Hz}$ crystals in Plasmodium food vacuoles play an important catalytic role in heme crystallization process (Pisciotta et al., in press).

In conclusion, heme crystallization has appeared independently in several groups of blood-feeding organisms that have no common ancestor. Among triatomine bugs, however, the ability to make $\mathrm{Hz}$ crystals seems to be generalized and can be regarded as the hallmark of the adaptation of the Triatominae to deal with heme intrinsic toxicity.

\section{Acknowledgements}

We would like to thank Prof. José Jurberg, from Laboratório Nacional e Internacional de Referência em Taxonomia de Triatomíneos at Dept. Entomologia, Fiocruz, Rio de Janeiro for kindly supplying blood-fed triatomines utilized in the present study. We are also indebted to Mr. Cláudio Figueiras, Mr. José de S. L. Junior and Mrs. Litiane M. Rodrigues for the excellent technical support. Supported by TWAS, CNPq, FAPERJ, FUJB, FAPESB, FINEP, PRONEX, PADCT and Capes. MFO and $\mathrm{PLO}$ are research scholars of CNPq.

\section{References}

Bendrat, K., Berger, B.J., Cerami, A., 1995. Haem polymerization in malaria. Nature 378, 138-139.

Blauer, G., Akkawi, M., 2002. Alcohol-water as a novel medium for betahematin preparation. Arch. Biochem. Biophys. 398, 7-11.

Brindley, P.J., Kalinna, B.H., Dalton, J.P., Day, S.R., Wong, J.Y.M., Smythe, M.L., McManus, D.P., 1997. Proteolytic degradation of host hemoglobin by schistosomes. Mol. Biochem. Parasitol. 89, 1-9.

Chen, M.M., Shi, L., Sullivan Jr., D.J., 2001. Haemoproteus and Schistosoma synthesize haem polymers similar to Plasmodium hemozoin and betahematin. Mol. Biochem. Parasitol. 113, 1-8.

Chou, A.C., Fitch, C.D., 1980. Hemolysis of mouse erythrocytes by ferriprotoporphyrin IX and chloroquine. J. Clin. Invest. 66, 856-858.

Dansa-Petretski, M., Ribeiro, J.M., Atella, G.C., Masuda, H., Oliveira, P.L., 1995. Antioxidant role of Rhodnius prolixus heme-binding protein. Protection against heme-induced lipid peroxidation. J. Biol. Chem. 270, 10893-10896.

Davies, M.J., 1988. Detection of peroxyl and alkoxyl radicals produced by reaction of hydroperoxides with heme-proteins by electron spin resonance spectroscopy. Biochim. Biophys. Acta 964, 28-35.

Dorn, A., Stoffel, R., Matile, H., Bubendorf, A., Ridley, R.G., 1995. Malarial haemozoin/ $\beta$-haematin supports haem polymerization in the absence of protein. Nature 374, 269-271

Dorn, A., Vippagunta, S.R., Matile, H., Bubendorf, A., Vennerstrom, J.L., Ridley, R.G., 1998. A comparison and analysis of several ways to promote haematin (haem) polymerisation and an assessment of its initiation in vitro. Biochem. Pharmacol. 55, 737-747.

Egan, T.J., 2002. Physico-chemical aspects of hemozoin (malaria pigment) structure and formation. J. Inorg. Biochem. 91, 19-26.

Egan, T.J., Ross, D.C., Adams, P.A., 1994. Quinoline anti-malarial drugs inhibit spontaneous formation of $\beta$-haematin (malaria pigment). FEBS Lett. 352, $54-57$.

Egan, T.J., Mavuso, W.M., Ncokazi, K.K., 2001. The mechanism of $\beta$-hematin formation in acetate solution. Parallels between hemozoin formation and biomineralization processes. Biochemistry 40, 204-213.

Egan, T.J., Combrinck, J.M., Egan, J., Hearne, G.R., Marques, H.M., Ntenteni, S., Sewell, T., Smith, P.J., Taylor, D., Van Schalkwyk, D.A., Walden, J.C., 2002. Fate of haem iron in the malaria parasite Plasmodium falciparum. Biochem. J. 365, 343-347.

Egan, T.J., Chen, J.Y., de Villiers, K.A., Mabotha, T.E., Naidoo, K.J., Ncokazi, K.K., Langford, S.J., McNaughton, D., Pandiancherri, S., Wood, B.R., 2006.
Haemozoin (beta-haematin) biomineralization occurs by self-assembly near the lipid/water interface. FEBS Lett. 80, 5105-5110.

Fitch, C.D., Kanjananggulpan, P., 1987. The state of ferriprotoporphyrin IX in malaria pigment. J. Biol. Chem. 262, 15552-15555.

Fitch, C.D., Cai, G.Z., Chen, Y.F., Shoemaker, J.D., 1999. Involvement of lipids in ferriprotoporphyrin IX polymerization in malaria. Biochim. Biophys. Acta 1454, 31-37.

Francis, S.E., Sullivan Jr., D.J., Goldberg, D.E., 1997. Hemoglobin metabolism in the malaria parasite Plasmodium falciparum. Annu. Rev. Microbiol. 51, $97-123$

Gaunt, M., Miles, M., 2000. The ecotopes and the evolution of Triatomine bugs (Triatominae) and their associated Trypanosomes. Mem. Inst. Oswaldo Cruz 95, 557-565.

Graça-Souza, A.V., Maya-Monteiro, C., Braz, G.R.C., Paes, M.C., Sorgine, M.H.F., Oliveira, M.F., Oliveira, P.L., 2006. Adaptations against heme toxicity in blood feeding arthropods. Insect Biochem. Mol. Biol. 36, 322-335.

Gutierrez, L.S., Burgos, M.H., 1986. The surface extracellular coat of the midgut in Triatoma infestans. I. Mechanism of development. J. Ultrastruct. Mol. Struct. Res. 95, 75-83.

Hempelmann, E., Motta, C., Hughes, R., Ward, S.A., Bray, P.G., 2003. Plasmodium falciparum: sacrificing membrane to grow crystals? Trends Parasitol. 19, 23-26.

Jackson, K.E., Klonis, N., Ferguson, D.J., Adisa, A., Dogovski, C., Tilley, L., 2004. Food vacuole-associated lipid bodies and heterogeneous lipid environments in the malaria parasite, Plasmodium falciparum. Mol. Microbiol. 54, 109-122.

Lane, N.J., Harrison, J.B., 1979. An unusual cell surface modification: a double plasma membrane. J. Cell Sci. 39, 355-372.

Lowry, H.O., Rosebrough, N.J., Farr, A.L., Randall, R.J., 1951. Protein measurement with the Folin phenol reagent. J. Biol. Chem. 193, 265-275.

Maya-Monteiro, C.M., Alves, L.R., Pinhal, N., Abdalla, D.S., Oliveira, P.L., 2004. HeLp, a heme-transporting lipoprotein with an antioxidant role. Insect Biochem. Mol. Biol. 34, 81-88.

Oliveira, M.F., Silva, J.R., Dansa-Petretski, M., de Souza, W., Lins, U., Braga, C.M.S., Masuda, H., Oliveira, P.L., 1999. Haem detoxification by an insect. Nature 400, 517-518.

Oliveira, M.F., Silva, J.R., Dansa-Petretski, M., de Souza, W., Braga, C.M.S., Masuda, H., Oliveira, P.L., 2000a. Haemozoin formation in the midgut of the blood sucking insect Rhodnius prolixus. FEBS Lett. 477, 95-98.

Oliveira, M.F., d'Avila, J.C.P., Torres, C.R., Oliveira, P.L., Tempone, A.J., Rumjanek, F.D., Silva, J.R., Dansa-Petretski, M., Oliveira, M.A., de Souza, W., Braga, C.M.S., Ferreira, S.T., 2000b. Haemozoin in Schistosoma mansoni. Mol. Biochem. Parasitol. 111, 217-221.

Oliveira, M.F., Timm, B.L., Machado, E.A., Miranda, K., Attias, M., Silva, J.R., Dansa-Petretski, M., de Oliveira, M.A, de Souza, W., Pinhal, N.M., Sousa, J.J., Vugman, N.V., Oliveira, P.L., 2002. On the pro-oxidant effects of haemozoin. FEBS Lett. 512, 139-144

Oliveira, M.F., d'Avila, J.C.P., Tempone, A.J., Soares, J.B.R., Rumjanek, F.D., Ferreira-Pereira, A., Ferreira, S.T., Oliveira, P.L., 2004. Inhibition of heme aggregation by chloroquine reduces Schistosoma mansoni infection. J. Infect. Dis. 190, 843-852.

Oliveira, M.F., Kycia, S.W., Gómez, A., Kosar, A.J., Bohle, D.S., Hempelmann, E., Menezes, D., Vannier-Santos, M.A., Oliveira, P.L., Ferreira, S.T., 2005. Structural and morphological characterization of hemozoin produced by Schistosoma mansoni and Rhodnius prolixus. FEBS Lett. 579, 6010-6016.

Orjih, A.U., Banyal, H.S., Chevli, R., Fitch, C.D., 1981. Hemin lyses malaria parasites. Science 214, 667-669.

Pagola, S., Stephens, P.W., Bohle, D.S., Kosar, A.D., Madsen, S.K., 2000. The structure of malaria pigment $\beta$-haematin. Nature 404, 307-310.

Paiva-Silva, G.O., Cruz-Oliveira, C., Nakayasu, E.S., Maya-Monteiro, C.M., Dunkov, B.C., Masuda, H., Almeida, I.C., Oliveira, P.L., 2006. A hemedegradation pathway in a blood-sucking insect. Proc. Natl. Acad. Sci. U. S. A. 103, 8030-8035.

Pisciotta, J.M., Ponder, E.L., Fried, B., Sullivan, D., 2005. Hemozoin formation in Echinostoma trivolvis rediae. Int. J. Parasitol. 35, 1037-1042.

Pisciotta, J.M., Coppens, I., Tripathi, A.K., Scholl, P.F., Shuman, J., Bajad, S., Shulaev, V., Sullivan, D.J., in press. The role of neutral lipid nanospheres in Plasmodium falciparum heme crystallization. Biochem. J. 
Ryter, S.W., Tyrrel, R.M., 2000. The heme synthesis and degradation pathways: Role in oxidant sensitivity. Heme oxygenase has both pro- and antioxidant properties. Free Radic. Biol. Med. 28, 289-309.

Schmitt, T.H., Frezzati Jr., W.A., Schreier, S., 1993. Hemin-induced lipid membrane disorder and increased permeability: a molecular model for the mechanism of cell lysis. Arch. Biochem. Biophys. 307, 96-103.

Schofield, C.J., 2000. Trypanosoma cruzi - the vector-parasite paradox. Mem. Inst. Oswaldo Cruz 95, 535-544.

Slater, A.F.G., Cerami, A., 1992. Inhibition by Chloroquine of a novel haem polymerase enzyme activity in malaria trophozoites. Nature 355, 167-169.

Slater, A.F.G., Swiggard, W.J., Orton, B.R., Flitter, W.D., Goldberg, D.E., Cerami, A., Henderson, G.B., 1991. An iron carboxylate bond links the heme units of malaria pigment. Proc. Natl. Acad. Sci. U. S. A. 88, 325-329.
Sullivan, D.J., 2002. Theories on malarial pigment formation and quinoline action. Int. J. Parasitol. 32, 1645-1653.

Sullivan, D.J., Gluzman, I.Y., Goldberg, D.E., 1996. Plasmodium hemozoin formation mediated by histidine-rich proteins. Science 271, 219-221.

Tappel, A.L., 1955. Unsaturated lipide oxidation catalyzed by hematin compounds. J. Biol. Chem. 217, 721-733.

Van der Zee, J., Barr, D.P., Mason, R.P., 1996. ESR spin trapping investigation of radical formation from the reaction between hematin and tert-butyl hydroperoxide. Free Radic. Biol. Med. 20, 199-206.

Vincent, S.H., 1989. Oxidative effects of heme and porphyrins on proteins and lipids. Semin. Hematol. 26, 105-113. 